\title{
Development of portable measuring system for testing of electrical vehicle's heat energy recovery system
}

\author{
Kornél Sarvajcz \\ Department of Electrical and Electronic Engineering \\ University of Debrecen \\ Debrecen, Hungary \\ sarvajcz@science.unideb.hu
}

\author{
Angela Váradiné Szarka \\ Department of Electrical and Electronic Engineering \\ University of Debrecen \\ Debrecen, Hungary \\ angela.varadi@science.unideb.hu
}

\begin{abstract}
Nowadays the consumer society applies a huge amount of energy in many fields including the transportation sector. The application of the internal combustion vehicles contribute substantially to the air pollution. An alternative solution for reducing energy consumption is replacing the internal combustion vehicles by electrical or hybrid vehicles. Today one of the biggest disadvantages of the electrical vehicles is the finite capacity of batteries. The research topic presented in this paper is the „Energy Harvesting”, and development of energy recovery system for electrical vehicles which largely contributes in increasing the driving range. At the current phase of the research efficiency analysis of the heat energy recovery devices are investigated in real driving circumstances. Computer based mobile and wireless measurement system for the analysis was developed, tested and installed in a real vehicle. Driving tests were performed and analysed in different circumstances.
\end{abstract}

Keywords-Energy Harvesting, Thermoelectric generator, Energy recovery system, electrical vehicle

\section{INTRODUCTION}

In this research we examine the methods of Energy Harvesting, which contributes the reducing of energy consumption's losses. The research focuses on the investigation and development of energy recovery systems in electric vehicles. In this paper the authors show in detailed the operation of thermoelectric devices furthermore also includes detailed information about development of a new calibration and test system for TEGs. This system is able to analyse thermoelectric processes applying different semiconductor elements and changing temperature difference between the cold and hot points in range of 0 and $400{ }^{\circ} \mathrm{C}$. The paper introduces operation point measurement of different types TEGs showing its maximum extractable electrical output power. The paper also contains detailed examination of measurement system developed for energy recovery, which can be applied in hybrid and electrical vehicles. The measuring system is able to detect two temperatures, current and voltage of TEG, GPS data and dynamic data of vehicle in the same time. The measuring data are sent via wireless communication to cloud based storage.

\section{METHODOLOGY}

Energy harvesting methods and equipment provide energy recycling processes suitable for saving, storing and using energy losses at a later time for specially defined and limited applications. The main question in such system is what will be, what can be the energy retrieving and transferring medium? The theoretical answer is all energy sources suitable for using with some active sensing system can be suitable, which means that if we can define an active sensor for the actual energy source, providing electrical output signal without power supply, depending its power strength capacity it can be used for energy harvesting purposes. The main problem, that these output signals in most of cases have extremely low power limiting its use for energy harvesting. So we should select such systems where the operation of actuatorsensor system provides high enough energy losses for harvesting. The most obvious solution is to use mechanical energy sources, magnetic induction energy transformers using vibration, rotation, flow- or press change are well known. Probably the most widely used method for harvesting is conversion of the radiative energy. The light including infrared, ultraviolet and visible ranges, radiofrequency, airwaves, thermal radiation provide basic concepts for such systems using light elements, antennas, thermo- and piezo cells.

TABLE I. Comparison of energy harvesting methods [1] 


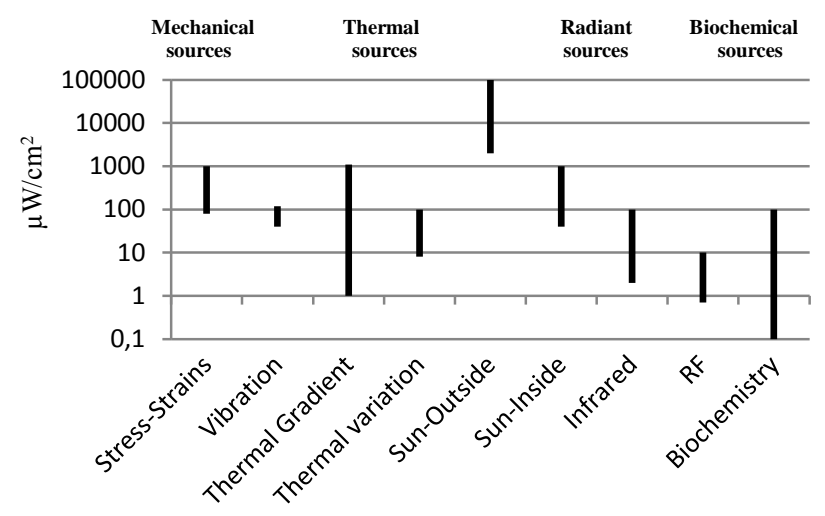

\section{THERMOELECTRIC GENERATOR}

The largest part of electrical losses is dissipated in form of heat, electrical and electronic producers spend huge resources to develop ideal cooling systems for electrical equipment. As thermoelectric generators use temperature difference for operation besides of energy harvesting it has an additional advantage which is the cooling effect. Our research group has developed a system suitable for diagnostic and evaluation of different TEGs. The system is presented in details in final paper.

TEGs have three main parts: N-type semiconductor, P-type semiconductor, and also a copper conductor providing contact surface between semiconductors. The most commonly used semiconductor is Bismuth Telluride $\left(\mathrm{Bi}_{2} \mathrm{Te}_{3}\right)$. The element is installed between two insulating surfaces made of ceramic material $\left(\mathrm{Al}_{2} \mathrm{O}_{3}\right)$ in most of cases. Theory of operation of TEGs is based on thermoelectric phenomena and processes which can be described by Seebeck, Peltier and Thomson effects.[2,3,4,5]

\section{CALIBRATION SYSTEM FOR THERMOELECTRIC GENERATORS}

Validation of TEG modules of different types and sizes require a calibration system of high reliability and repeatability therefore the research group decided to develop a calibration system for our special needs.

Temperature of the heated side is generated by a $450 \mathrm{~W}$ Infrared Top Ceramic Heater. It can generate $750{ }^{\circ} \mathrm{C}$ temperature; therefore housing with special heat insulation should be used. The house is made of Bakelite plate with width of $15 \mathrm{~mm}$. The Bakelite is one of the best heat insulating material with $0.23[\mathrm{~W} / \mathrm{mK}]$ heat conductivity coefficient. Inside of the Bakelite housing a secondary insulation is applied which made of a special non-combustible asbestos alloy. This material is used for inner insulation of combustion chamber in household furnaces. Between the heater piece and TEG an aluminium inner spreader is included in order to provide equal temperature on the surface of the TEG. As there are many different TEG sizes are available from different producers, modular aluminium spacers are designed for TEGs. These spacers are used for flexible assembling and insertion of $\mathrm{K}$ type thermocouples measuring temperature of hot point.
The spacer also ensures the homogeneous temperature on the full surface of TEG's.

For cooling of the cold point a flow water cooler is used, which is connected to household water tube. Also an aluminium spacer measuring temperature of cold point is inserted between the cooler and TEG module. Aluminium spacers are designed for all standard sized TEGs in order to ensure homogeneous temperature distribution on the full surface.
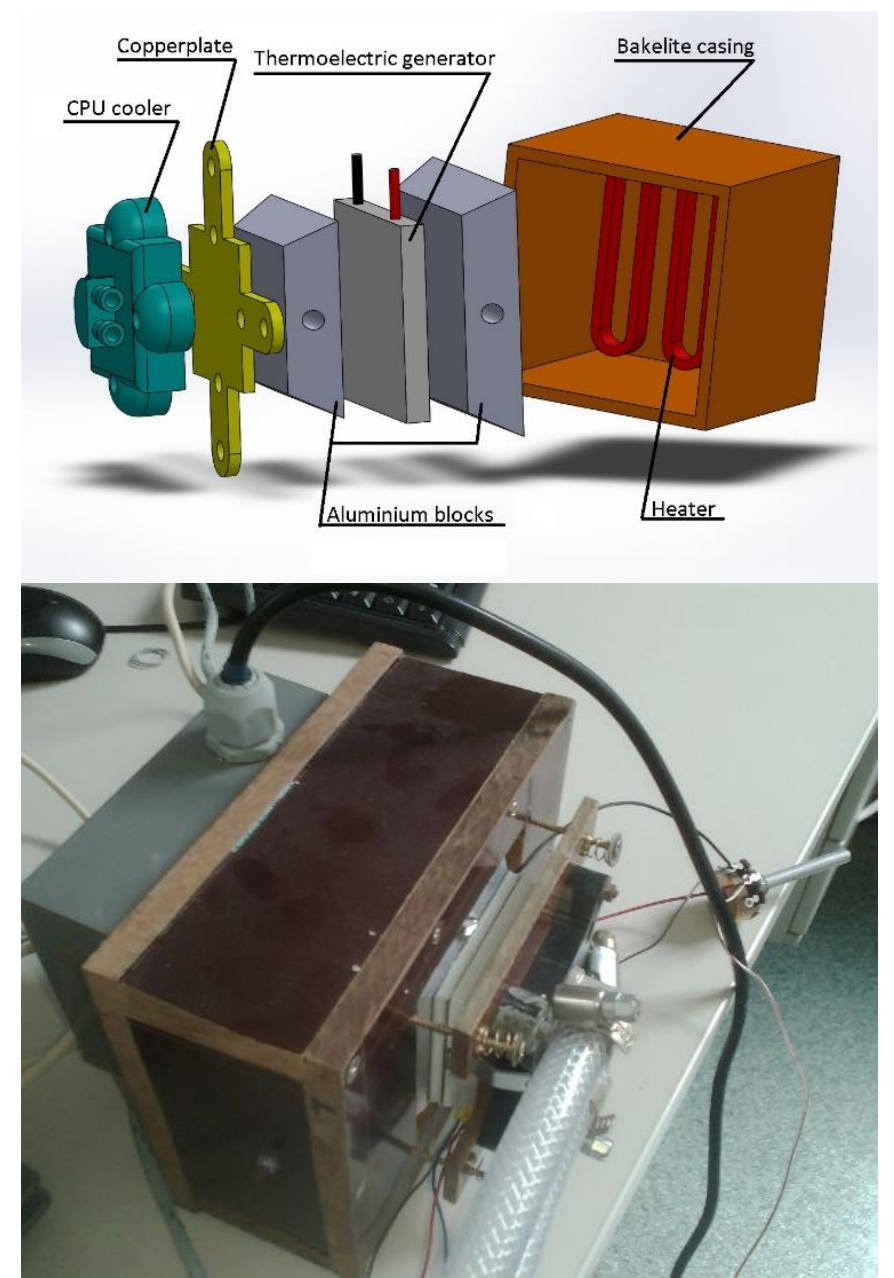

Fig. 1. Measuring and calibrating equipment

Control of tests are developed using National Instruments hardware and software. It will be expounded in detail in full paper. 


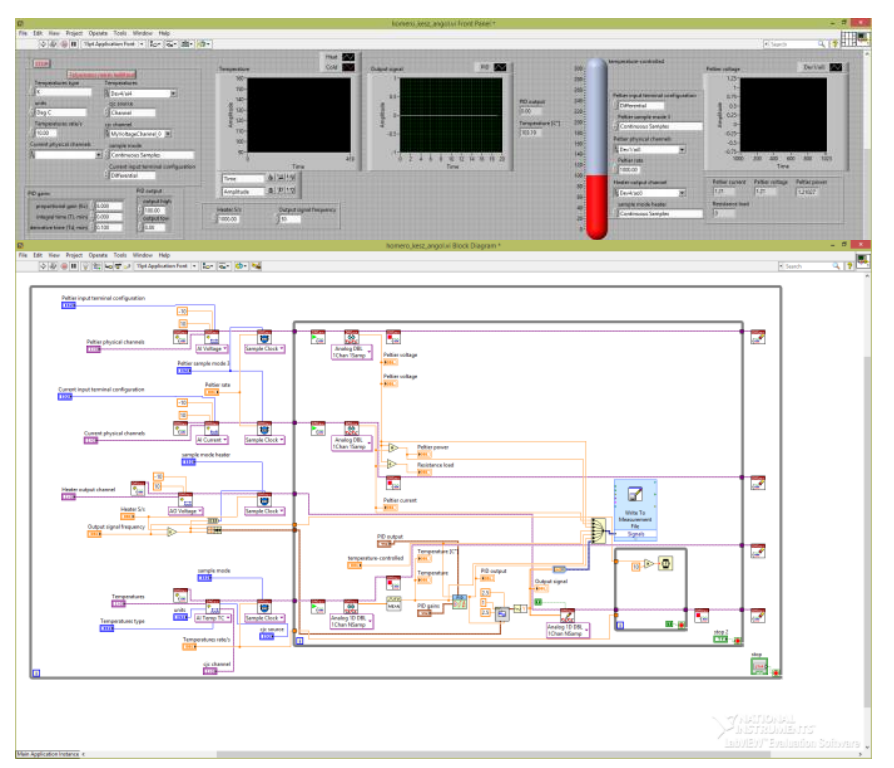

Fig. 2. Measuring and control software

In reference [6] the system presented particularly.

\section{RESUlTS}

The operating point tests were done with two different types TEG module, which are originated from the same manufacturer. The examined TEG modules have the following serial numbers: the Custom Thermoelectric Inc. 2411G-7L3115CX1 and 1261G-7L31-24CX1. During the measurements, applying the internally developed calibrating equipment eight different temperature differences were set and the values of the load resistance were set by $0.1 \Omega$ steps, in the $0-5 \Omega$ range. The aim of our examination was determination the maximum power of TEG modules at different temperature differences. Based on Figure 4 and Figure 5 can be determined that the extractable electric power is $2.8 \mathrm{~W}$ in case of the $200{ }^{\circ} \mathrm{C}$ temperature difference and $0.002809 \mathrm{~m}^{2}$ surface.

$1261 G-7 L 31-24 C X 1$

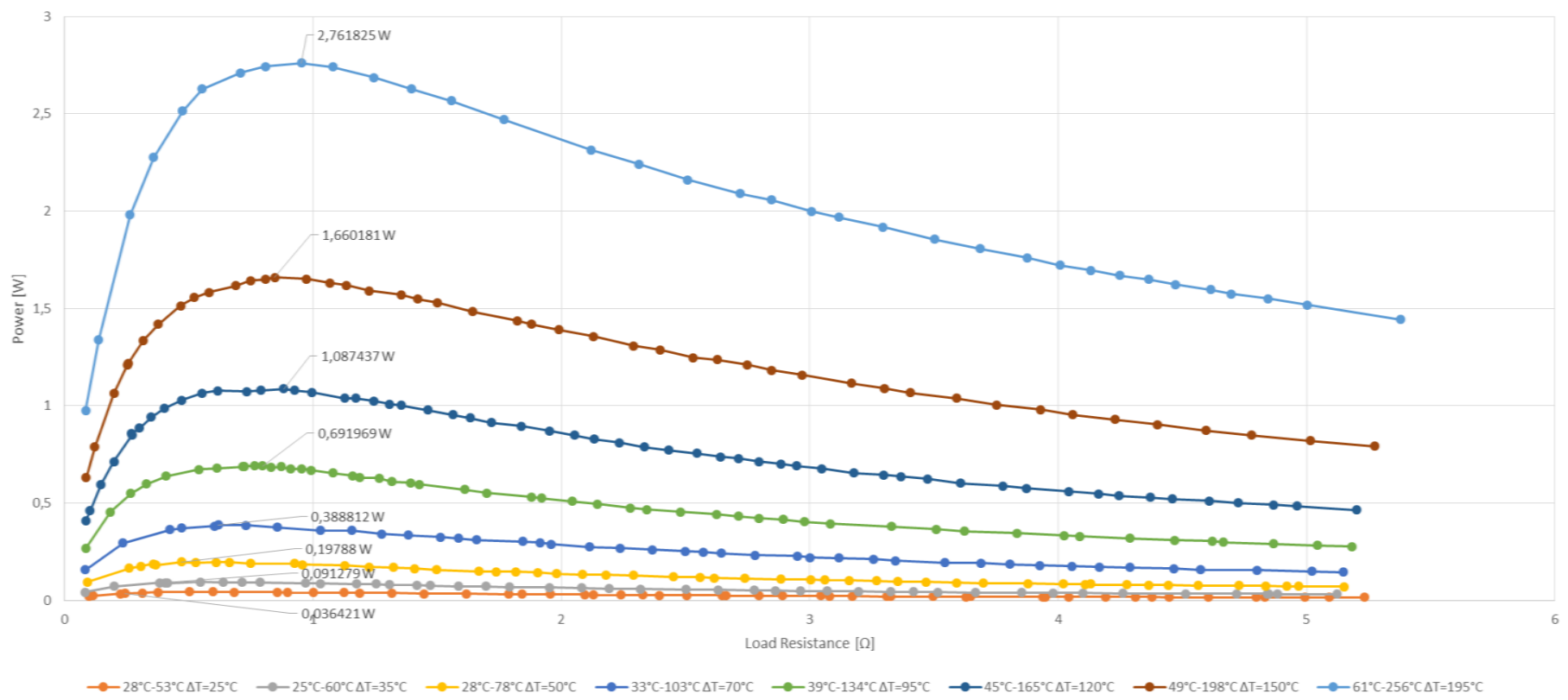

Fig. 3. 1261G-7L31-24CX1 TEG module operating point measurements 


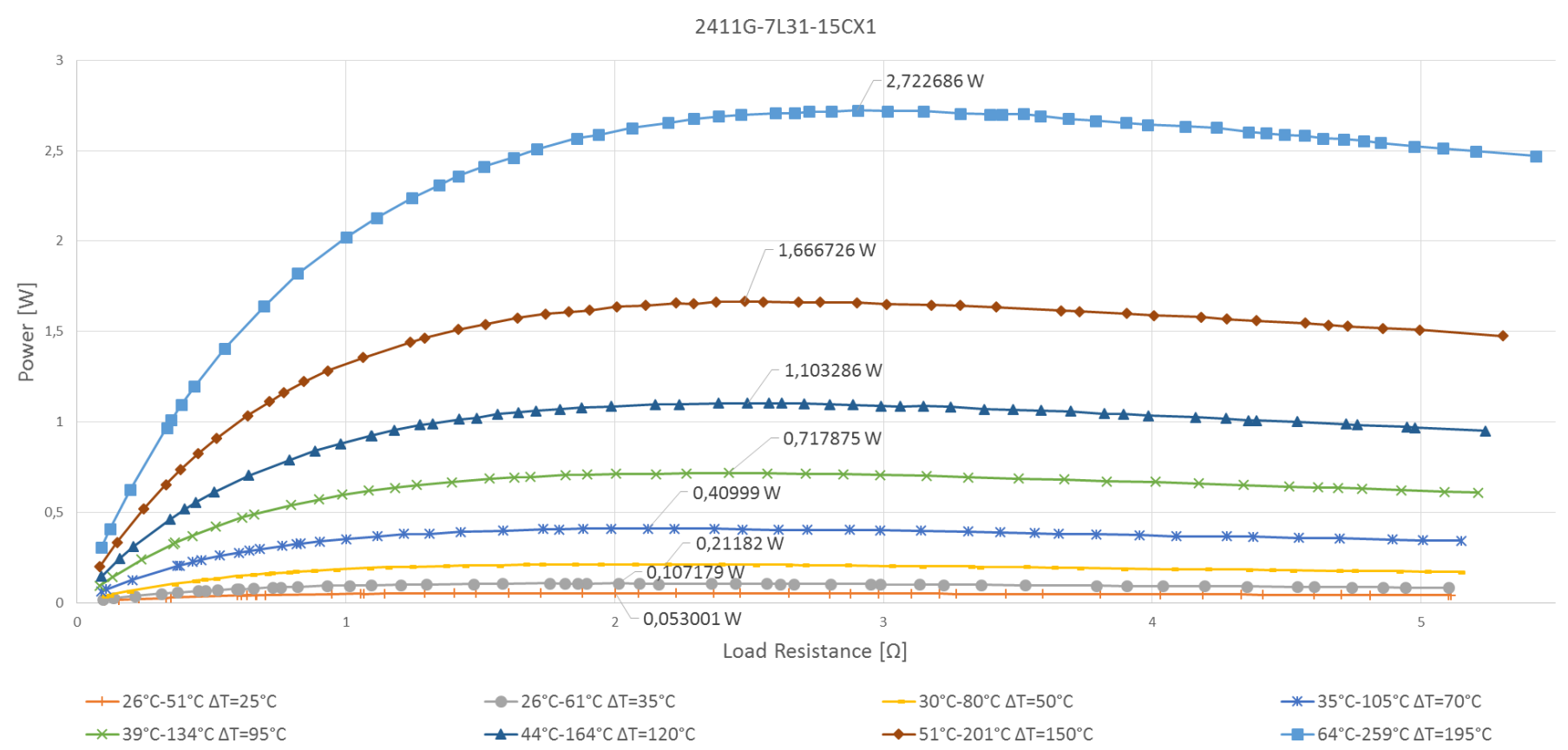

Fig. 4. 2411G-7L31-15CX1 TEG module operating point measurements

Based on Fig. 3 can be determined that the TEG module's internal resistance is temperature-dependent and it changes about approximately between $0.4-0.8 \Omega$. The maximum power can be extracted from the TEG, when the internal resistance's value and load resistance's value are the same. The internal resistance of the other TEG module, which is illustrated on Fig. 4 is also temperature-dependent, but it covers other ranges. It changes between 2-3 $\Omega$. For the most optimal operation of the heat energy recovery the harmonization of internal resistance's value and load resistance's value is necessary.

\section{CONCLUSION}

The introduced operating point measurements testify that the TEG is applicable device of the Energy Harvesting. In this paper introduced TEG simulation and the calibrating system contribute largely to the basic research. In an electrical vehicle the average operating temperature of electrical equipment is approximately $80^{\circ} \mathrm{C}$, and it does not require additional energy for reach this temperature. Due to the transportation the wind as cooling agent is available. The current concepts of the vehicles additional energy are necessary for cooling the electronics and the electromotor. The application of TEGs is suitable for energy generation using heat loss and is also suitable for saving of the traditional cooling system's energy requirements. The result of the current research confirms that TEGs application can be a significant part of the electrical vehicle's energy recovery system. In this research's current phase the TEG module is installed on a vehicle, and the research team test it during several temperature difference.

\section{REFERENCES}

[1] S. Boisseau, G. Despesse and B. Ahmed Seddik,2012.: Electrostatic Conversion for Vibration Energy Harvesting, Small-Scale Energy Harvesting, ISBN 978-953-51-0826-9

[2] L. Chena, J. Gonga, F. Suna, C. Wub,2002.: Effect of heat transfer on the performance of thermoelectric generators, International Journal of Thermal Sciences Volume 41, Pages 95-99

[3] J, E. Matthews 2011.: Thermoelectric and heat flow phenomena in mesoscopic systems, Doctor of Philosophy DISSERTATION

[4] D. Yan 2011.: Modeling and Application of a Thermoelectric Generator, Masters of Applied Science Thesis, University of Toronto

[5] S. Nieswand 2011.: A Peltier cooling system for SiPM temperature stabilization, Masters of Applied Science Thesis, University of Toronto

[6] K. Sarvajcz, A. Szarka 2015.: SIMULATION AND CALIBRATION TEST OF THERMOELECTRIC GENERATORS, XXI IMEKO World Congress Measurement in Research and Industry, Prague, Czech Republic 